\title{
Original
}

\section{Influence of acute high-intensity exercise on salivary nitric oxide levels}

\author{
Ryota Sone1), Nobuhiko Eda'2), Keisei Kosaki³), Momoko Endo4), \\ and Koichi Watanabe ${ }^{3)}$
1)Graduate School of Comprehensive Human Sciences, University of Tsukuba, Tsukuba, Japan
2)Faculty of Sports Sciences, Waseda University, Tokorozawa, Japan
3)Faculty of Health and Sport Sciences, University of Tsukuba, Tsukuba, Japan.
4)Shinkamaekimae Orthopedic Clinic, Kamagaya, Japan

(Received June 18, 2018; Accepted September 18, 2018)

\begin{abstract}
This study, employing an exercise versus control crossover design, was conducted to investigate the influence of acute high-intensity exercise on salivary nitric oxide (NO) levels. Nine healthy males (aged 23.8 \pm 1.4 years) performed ergometer exercise at $80 \% \dot{\mathrm{V}} \mathrm{O}_{2 \text { peak }}$ for $60 \mathrm{~min}$, whereas controls sat at rest for $60 \mathrm{~min}$. Saliva samples were collected before (Pre: $0800 \mathrm{~h}$ ) and after (Post 0-h: 0900 h, Post 1-h: 1000 h, Post 2-h: 1100 h, Post 3-h: 1200 h) the interventions. Salivary NO levels were determined by colorimetric assay. It was found that the salivary NO levels in controls were decreased $(P<0.05)$ at Post 0-h $(-94$ $\pm 15)$, Post 1-h $(-80 \pm 20)$, Post 2-h $(-92 \pm 34)$ and Post 3-h $(-145 \pm 39)$ relative to the Pre values. Under exercise conditions, salivary NO levels did not change after high-intensity ergometer exercise relative to the Pre values. Thus, the response of salivary NO levels appeared to differ between high-intensity ergometer exercise and inactivity, that exercise-related stress induces the production of salivary NO.
\end{abstract}

Keywords: salivary nitric oxide; high-intensity exercise; oral immunity.

Correspondence to Dr. Koichi Watanabe, Faculty of Health and Sport Science, University of Tsukuba, 1-1-1 Tennodai, Tsukuba, Ibaraki 305-8574, Japan

Fax: +81-29-853-5902

E-mail: watanabe.koichi.ga@u.tsukuba.ac.jp

doi.org/10.2334/josnusd.18-0205

DN/JST.JSTAGE/josnusd/18-0205

\section{Introduction}

Nitric oxide (NO) is synthesized from 1-arginine by nitric oxide synthase (NOS), which has three isoforms: neuronal NOS (nNOS or NOS1), inducible NOS (iNOS or NOS2) and endothelial NOS (eNOS or NOS3) (1). Among these NOS isoforms, iNOS is reported to be induced by lipopolysaccharide and various cytokines secreted by macrophages (2). In humans, iNOS is reported to be expressed at a significantly higher level in diseased that in healthy periodontal tissues (3). In addition, NO is associated with nonspecific immunity, and is produced in large quantities during host defense and immunologic reactions (4). Excessive production of NO in the salivary glands is reportedly associated with the development of various diseases, such as Sjögren's syndrome $(5,6)$. NO generated by activated macrophages has cytostatic properties (7) and it is likely to have a role in nonspecific immunity (8). Thus, salivary NO may reflect nonspecific immunological reactions in the oral cavity.

Repetitive intensive exercise has been reported to induce a number of disorders such as infections, overtraining syndrome, and chronic fatigue syndrome (9). Salivary secretory immunoglobulin A (SIgA) plays a role in specific immunity against upper respiratory tract infections (URTIs). Intense exercise reduces SIgA levels (10) for up to two hours after exercise (11). Low levels of SIgA are associated with a high risk of URTIs (12-14). As an index for monitoring physical condition, differences in SIgA and NO levels in saliva may indicate specific and nonspecific immunological reactions. 
Salivary NO levels are reported to increase during and after exercise $(4,15)$, and also to vary with training intensity and load (16). However, the influence of exercise that leads to a decline of immune function on salivary NO level is still unknown. On the other hand, SIgA has an important role in the prevention of acute illnesses, including URTIs in the oral cavity. Assessment of changes in both salivary NO and SIgA levels during and after exercise might therefore help to clarify the immune response to exercise-related stress. Although previous studies have reported that salivary NO levels increase as a result of exercise $(4,15)$, no previous study has investigated this issue using a random crossover design in subjects at rest. As some saliva components show diurnal variation $(17,18)$, it is necessary to considered changes at rest when assessing the influence of acute exercise.

The differences in the response of salivary NO levels to resting and exercise conditions remain unknown. In order to investigate this issue, the present study using a random crossover design employed both high-intensity exercise and resting conditions. It was hypothesized that the results of this study might help to clarify whether measurement of salivary NO would be useful in the management of medical conditions.

\section{Subjects}

\section{Materials and Methods}

Nine healthy male subjects volunteered to participate in this interventional study (Table 1). The eligibility criteria for the subjects were that none of them smoked, or had any diseases or injury. This study was approved by the Ethics Committee of the Faculty of Health and Sport Sciences of the University of Tsukuba (\#Tai 27-99, year 2015) and was conducted in accordance with the principles outlined in the Declaration of Helsinki, all subjects providing written informed consent before inclusion (19).

\section{Design}

Employing a crossover design, participants were evaluated under two conditions: sitting at rest (control) and while performing acute high-intensity exercise at $80 \%$ $\mathrm{VO}_{2 \text { peak }}$ with a bicycle ergometer (75XL3; Combi Wellness Co., Ltd, Tokyo, Japan). Under both conditions, subjects were instructed to refrain from alcohol, caffeine, smoking, and intense physical activity on the day before the start of the experiment up to three hours postintervention until sampling was completed. On the day of the experiment, all subjects arrived at the laboratory by $0730 \mathrm{~h}$ after an overnight fast. Before the interventions, subjects performed an incremental cycling test for measurement of peak oxygen consumption $\left(\mathrm{VO}_{2 \text { peak }}\right)$.
Table 1 Characteristics of subjects

\begin{tabular}{lc}
\hline Variable $(n=9)$ & Mean $\pm \mathrm{SD}$ \\
\hline Age $($ years $)$ & $23.8 \pm 1.4$ \\
Height $(\mathrm{cm})$ & $174.9 \pm 3.2$ \\
Body weight $(\mathrm{kg})$ & $68.4 \pm 5.8$ \\
Body mass index $\left(\mathrm{kg} / \mathrm{m}^{2}\right)$ & $22.3 \pm 1.8$ \\
$\mathrm{VO}_{2}$ peak $(\mathrm{mL} / \mathrm{kg} / \mathrm{min})$ & $49.0 \pm 4.6$ \\
$\mathrm{HRmax}$ during exercise $(\mathrm{bpm})$ & $183 \pm 12$ \\
\hline Values are mean $\pm \mathrm{SD}$ &
\end{tabular}

$\mathrm{VO}_{2 \text { peak }}$ : peak oxygen uptake per minute; HRmax: maximal heart rate.

During the test exercise, expired gases were collected via a facemask, and the oxygen uptake and carbon dioxide exhaled were calculated by the breath-by-breath method (AE310S; Minato Medical Science Co., Ltd, Osaka, Japan). The acute high-intensity exercise intervention was performed at least four days after the first exercise test, but within two weeks. After a 10-min warm-up, subjects cycled for $50 \mathrm{~min}$ at $80 \% \mathrm{VO}_{2 \text { peak }}$. During the intense exercise, the workload was controlled at a peak oxygen uptake of $80 \%$. Every 5 min during the exercise test, the subjects were asked for their rating of perceived exertion (RPE) using the Borg category scale (20).

\section{Sample collection}

Saliva samples were obtained before (Pre) and after the interventions and at every hour until $3 \mathrm{~h}$ after each intervention (Post 1-h, 2-h, and 3-h) for measurement of salivary NO levels, SIgA and total protein. Saliva samples were collected as described previously (21) after each participant's mouth had been rinsed thoroughly with distilled water. Saliva production was stimulated by chewing a sterile cotton swab (Salivette; Sarstedt Inc., Nümbrecht, Germany) at a frequency of $60 / 60 \mathrm{~s}$, and saliva was separated from the cotton swab by centrifugation at 3,000 rpm for $15 \mathrm{~min}$. After measurement of the sample volume (saliva flow rate), saliva samples were frozen at $-80^{\circ} \mathrm{C}$ and stored until the end of the analyses. Blood samples were collected for measurement of reactive oxygen metabolite-derived compounds (d-ROMs) from a fingertip into a $300-\mu \mathrm{L}$ micro tube (DI-002-06, Wismerll Co., Ltd., Tokyo, Japan) using a lancet (DI-003b, Wismerll Co., Ltd.) before and after each intervention (Pre, Post).

\section{Assay of saliva}

The salivary level of NO was assessed as the nitrate concentration using a commercially available colorimetric assay kit (Nitric Oxide detection kit \#ADI-917-010, Enzo Life Sciences, Inc., Farmingdale, NY, USA), in accordance with the manufacturer's instructions. Total 


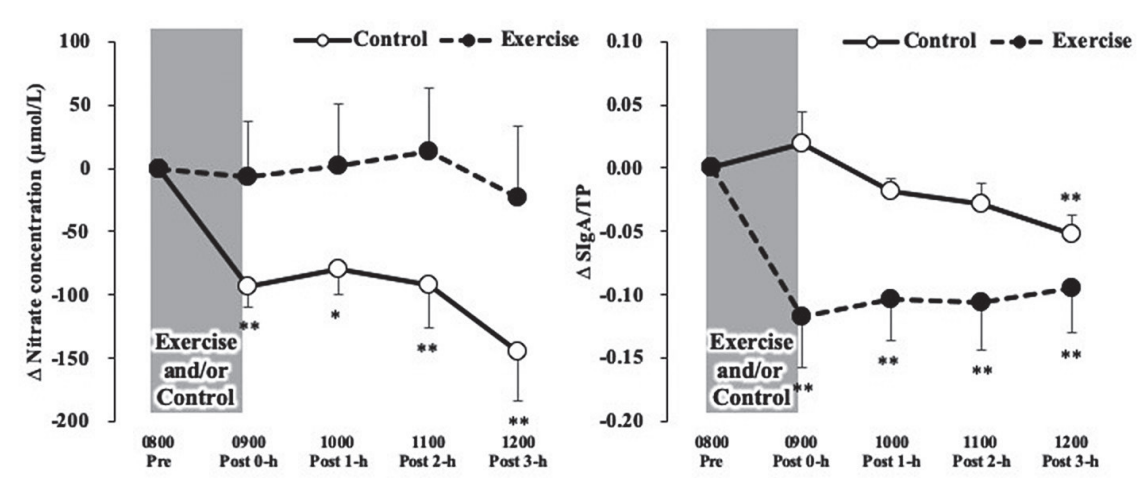

Fig. 1 Changes in $(\Delta)$ salivary nitrate concentration and SIgA corrected with total protein between $0800 \mathrm{~h}$ and $1200 \mathrm{~h}$ under exercise (black) and control (white) conditions. Values are presented as mean \pm SE. $* P<0.05$ Pre $(0800)$ vs. each Post $(0900,1000,1100,1200)$, $* * P<0.01$ Pre $(0800)$ vs. each Post $(0900,1000,1100,1200)$.

Table 2 Change of d-ROMs test, nitrate concentration, SIgA/total protein, SIgA concentration, total protein, saliva flow rate, and fatigue scale

\begin{tabular}{|c|c|c|c|c|c|c|c|}
\hline Variable $(n=9)$ & & $\begin{array}{l}0800 \mathrm{~h} \\
\text { Pre }\end{array}$ & $\begin{array}{l}0900 \mathrm{~h} \\
\text { Post } 0 \mathrm{~h}\end{array}$ & $\begin{array}{l}1000 \mathrm{~h} \\
\text { Post } 1 \mathrm{~h}\end{array}$ & $\begin{array}{l}1100 \mathrm{~h} \\
\text { Post } 2 \mathrm{~h}\end{array}$ & $\begin{array}{l}1200 \mathrm{~h} \\
\text { Post } 3 \mathrm{~h}\end{array}$ & Interaction \\
\hline \multirow{2}{*}{$\begin{array}{l}\text { d-ROMs test } \\
\text { (U.CARR) }\end{array}$} & Control & $278 \pm 12$ & $278 \pm 10$ & - & - & - & \multirow{2}{*}{0.019} \\
\hline & Exercise & $265 \pm 10$ & $289 \pm 13^{* *}$ & - & - & - & \\
\hline \multirow{2}{*}{$\begin{array}{l}\text { Nitrate concentration } \\
(\mu \mathrm{mol} / \mathrm{L})\end{array}$} & Control & $447 \pm 65$ & $353 \pm 57 * *$ & $367 \pm 56^{*}$ & $355 \pm 49 * *$ & $303 \pm 44 * *$ & \multirow{2}{*}{0.005} \\
\hline & Exercise & $388 \pm 82$ & $380 \pm 76$ & $389 \pm 66$ & $401 \pm 63$ & $365 \pm 53$ & \\
\hline \multirow[t]{2}{*}{ SIgA / total protein } & Control & $0.14 \pm 0.03$ & $0.16 \pm 0.04$ & $0.12 \pm 0.03$ & $0.11 \pm 0.03$ & $0.09 \pm 0.02 *$ & \multirow{2}{*}{0.000} \\
\hline & Exercise & $0.17 \pm 0.05$ & $0.06 \pm 0.01 * *$ & $0.07 \pm 0.02 * *$ & $0.07 \pm 0.01 * *$ & $0.08 \pm 0.02 * *$ & \\
\hline \multirow{2}{*}{$\begin{array}{l}\text { SIgA concentration } \\
(\mu \mathrm{g} / \mathrm{mL})\end{array}$} & Control & $43 \pm 13$ & $30 \pm 7$ & $29 \pm 6$ & $30 \pm 6$ & $26 \pm 3^{*}$ & \multirow{2}{*}{0.153} \\
\hline & Exercise & $33 \pm 8$ & $32 \pm 6$ & $22 \pm 4 *$ & $22 \pm 3^{*}$ & $29 \pm 6$ & \\
\hline \multirow{2}{*}{$\begin{array}{l}\text { Total protein } \\
(\mu \mathrm{g} / \mathrm{mL})\end{array}$} & Control & $341 \pm 70$ & $218 \pm 45$ & $285 \pm 56$ & $308 \pm 46$ & $380 \pm 80$ & \multirow{2}{*}{0.049} \\
\hline & Exercise & $220 \pm 31$ & $733 \pm 143^{* *}$ & $390 \pm 62$ & $380 \pm 63$ & $406 \pm 74$ & \\
\hline \multirow{2}{*}{$\begin{array}{l}\text { Saliva flow rate } \\
(\mathrm{mL} / \mathrm{min})\end{array}$} & Control & $1.381 \pm 0.316$ & $1.464 \pm 0.341$ & $1.492 \pm 0.358$ & $1.550 \pm 0.254$ & $1.449 \pm 0.246$ & \multirow{2}{*}{0.136} \\
\hline & Exercise & $1.268 \pm 0.285$ & $1.056 \pm 0.238$ & $1.354 \pm 0.308$ & $1.360 \pm 0.271$ & $1.397 \pm 0.253$ & \\
\hline \multirow{2}{*}{$\begin{array}{l}\text { Fatigue scale } \\
(\mathrm{mm})\end{array}$} & Control & $20 \pm 3$ & $20 \pm 3$ & $19 \pm 3$ & $17 \pm 3$ & $17 \pm 2$ & \multirow{2}{*}{0.000} \\
\hline & Exercise & $21 \pm 4$ & $76 \pm 7 * *$ & $43 \pm 6^{* *}$ & $36 \pm 6$ & $27 \pm 4$ & \\
\hline
\end{tabular}

protein in saliva was also measured by colorimetric assay (Pierce $660 \mathrm{~nm}$ Protein Assay kit \#22662, Thermo Fisher Scientific Inc., Waltham, MA, USA), in accordance with the manufacturer's instructions. The salivary SIgA concentration was measured by enzyme-linked immunosorbent assay (ELISA), as described by Akimoto et al. (21). All samples were assayed in duplicate, and the average of the two absorbance values was used as the representative value.

\section{Assay of blood}

d-ROMs were measured using a free radical and antioxidant potential determination device (Free Carrio Duo; Free Radical Elective Evaluator, Diacon International Co., Grosseto, Italy). In the d-ROMs test, the hydroperoxides in a $20-\mu \mathrm{L}$ blood sample react with a chromogen substrate at $37^{\circ} \mathrm{C}$ to yield a colored derivative, the intensity of which is directly proportional to the concentration of ROMs, in accordance with the Beer-Lambert law (22). This color intensity is quantified and the results are expressed in Caratelli Units (U.Carr.), where 1 U.Carr. is equivalent to $0.08 \mathrm{mg} / 100 \mathrm{~mL}$ hydrogen peroxide. The normal range for d-ROMs is between 250 and 300 U.Carr. (23).

\section{Statistical analysis}

Data were expressed as mean \pm SE. For all analyses, statistical significance was defined as $P<0.05$. The effects of the high-intensity exercise intervention on all outcome measures, except for d-ROMs, were subjected to two-way repeated (Time*Conditions) measures analysis of variance (ANOVA), followed by Dunnett's post hoc test. The d-ROMs were subjected to the Bonferroni post hoc test. All calculations were performed using SPSS software (ver. 24) (IBM Co., Armonk, NY, USA). 


\section{Results}

Figure 1 shows the changes in the subjects' salivary NO levels (nitrate concentration) and SIgA/total protein (SIgA/TP) before and after the control and exercise interventions. At the baseline (Pre) there were no significant differences in the salivary NO levels and SIgA/TP between the exercise and the control groups, whereas after the interventions significant differences were evident (interaction; Time*Condition: $P<0.01$ ). Under control conditions at rest, salivary NO levels were significantly decreased $(P<0.05)$ at Post 0 -h $(-94 \pm 15)$, Post 1 -h $(-80 \pm 20)$, Post $2-\mathrm{h}(-92 \pm 34)$ and Post $3-\mathrm{h}(-145 \pm 39)$ in comparison with Pre. Under exercise conditions, the $\mathrm{SIgA} / \mathrm{TP}$ levels were significantly decreased $(\mathrm{P}<0.01)$ at Post 0 -h $(-0.12 \pm 0.04)$, Post $1-\mathrm{h}(-0.10 \pm 0.03)$, Post 2 -h $(-0.11 \pm 0.04)$ and Post $3-\mathrm{h}(-0.09 \pm 0.04)$ in comparison with Pre.

Table 2 shows the changes in the values for d-ROMs, salivary NO levels, SIgA/TP, SIgA concentration, total protein, saliva flow rate, and the fatigue scale before and after the control and exercise interventions. Before the interventions, there were no significant differences in the values for d-ROMs, salivary NO levels, SIgA/TP, SIgA concentration, total protein, saliva flow rate, and the fatigue scale between the conditions. Significant interaction (Time*Condition) was observed for the d-ROMs value $(P<0.05)$, salivary NO levels $(P<0.01)$, SIgA/TP $(P<0.01)$, total protein $(P<0.01)$ and the fatigue scale $(P<0.01)$, whereas there was no significant interaction for the SIgA concentration and saliva flow rate. The post hoc test revealed a significant change in the d-ROMs value, SIgA/TP, SIgA concentration, total protein and fatigue scale under exercise conditions. On the other hand, salivary NO levels decreased significantly under the control conditions.

\section{Discussion}

The present study investigated the influence of acute high-intensity exercise on salivary NO levels using a crossover design involving exercise and control arms in healthy male subjects with a sedentary lifestyle. It was found that (1) under exercise conditions, salivary NO levels did not change significantly, and that (2) under control conditions, salivary NO levels showed a significant decrease. These results revealed a difference in the response of salivary NO to high-intensity exercise and sitting at rest.

Several previous studies have shown that the salivary NO level increases with exercise, but none of them compared the levels with those under control conditions at rest $(4,15)$. As the levels of several salivary components are reported to show a circadian rhythm $(17,18)$, the present study investigated differences in the salivary NO responses to control and exercise employing a random crossover design. Although high-intensity exercise did not elicit an increase in salivary NO levels, a significant decrease in salivary $\mathrm{NO}$ was observed under control conditions. These results partially support those of Gonzalez et al., who reported that salivary NO levels did not change after exercise (24). Another study has also reported that salivary NO levels were increased by exercise, although no comparison with a resting control was performed (15). Rahman et al. (15) employed Bruce's protocol, which is an "all-out" exercise regime inducing extreme fatigue within a short period. As previous studies did not employ a constant level of exercise difficulty, differences in the salivary NO response may have arisen. However, since salivary NO levels do show some fluctuation (Fig. 1), it can be assumed that differences in response to different interventions would occur.

The present study also demonstrated that salivary NO levels decreased under control conditions at rest. SIgA, which is a specific immune marker for URTIs, shows circadian variation $(17,18)$. The level of salivary alpha-amylase (sAA) activity also varies between morning and late afternoon $(17,18)$, and has been used as a stress indicator and/or biomarker for evaluation of immune function (25), as sAA has both digestive and antibacterial activity (26). Thus, the level of salivary NO may also show daytime changes similar to those of other salivary components. A previous study that examined the circadian rhythm of salivary nitrate and nitrite found that the level of nitrite rose at night (27). In the present study, the level of salivary NO was assessed as the salivary nitrate concentration, which may also have a circadian rhythm and increase at night, as both nitrate and nitrite are metabolites of salivary NO (28). In the present study, as the experiments were started early in the morning, the salivary NO levels may have reflected the increased concentration of nitrate accumulated during the night, as measured under resting conditions before the intervention. Considering this possibility, it might have been informative to have asked the subjects about their sleeping time using a questionnaire. The role of NO in the oral cavity as an antimicrobial reagent has been discussed $(29,30)$. It is well known that many bacteria are present in human saliva upon waking. Therefore, the decrease of salivary NO observed under control conditions may have been attributable to its active antibacterial action in the oral cavity during the morning.

This is the first study to have investigated the response of salivary NO to high-intensity exercise, which non- 
specifically attenuates immune function, as reflected in a decreased SIgA level. Based on data from a previous study (31), the intensity of the exercise was set at a level known to induce a decrease in SIgA, and indeed a corresponding decline in SIgA due to the exercise was observed. Furthermore, the data obtained using a visual analog scale (VAS) to assess the subjective degree of fatigue and d-ROMs as an index of oxidative stress indicated that exercise-related stress had indeed been induced. The most important finding of this study was that salivary $\mathrm{NO}$ appeared to be affected by high-intensity exercise, thus decreasing the level of immune function. The low level of SIgA found in this study is known to be related to a high risk of URTIs (12-14). Although the level of SIgA may be associated with general suppression of immune function, the possibility that NO secretion is more directly responsible for preventing infections at the early stage of pathogen contact has been reported (32). The expression of IgA has also been shown to be dependent on iNOS activity (33) in dendritic and T cells (34). NO activity may be a factor that impacts a range of aspects of innate mucosal immunity (35). Hence, situations associated with a high risk of developing URTIs may be characterized by a change in salivary NO levels. As this study did not investigate URTIs' symptoms, a further detailed study of the association between salivary NO levels and diseases including URTIs in the context of oral health will be necessary. In addition, it has been reported that high salivary NO levels are associated with the development of various diseases $(5,6)$. Although fractional exhaled $\mathrm{NO}(\mathrm{FeNO})$ has a reference range for respiratory diseases such as asthma, salivary NO has no reference value for diseases including those affecting the oral region. Therefore, it will also be necessary to set a cut-off value for salivary NO in the context of disease.

Although the Pre levels of salivary NO appeared to differ between the two conditions, the difference was not statistically significant. One limitation of this study was that the meals taken by the subjects on the day before the experiment were not regulated, and thus differed among individual. All of the study subjects were asked to refrain from ingesting anything except mineral water from 2100 $\mathrm{h}$ on the night before the experiment. Additionally, the subjects were instructed to eat their usual meals, as salivary NO, especially salivary nitrate and nitrite, is known to be affected by diet (36). Therefore, future studies of changes in salivary NO level in response to exercise will need to employ a precisely controlled diet.

In conclusion, the data obtained in this crossover study indicate that the response of salivary NO differs between high-intensity ergometer exercise and control conditions at rest in healthy male subjects with a sedentary lifestyle. The level of salivary NO did not change with exercise, but was decreased under control conditions. These findings suggest that salivary NO may be affected by exercise-related stress.

\section{Acknowledgments}

We thank Kai Matsuba, Tomoko Imai, Hiroshi Kumagai, and Hironaga Ito for their technical assistance.

\section{Conflict of interest}

The authors declare that they have no conflict of interest.

\section{References}

1. Nathan C, Xie QW (1994) Nitric oxide synthases: roles, tools, and controls. Cell 78, 915-918.

2. Xie QW, Cho HJ, Calaycay J, Mumford RA, Swiderek KM, Lee TD et al. (1992) Cloning and characterization of inducible nitric oxide synthase from mouse macrophages. Science 256, 225-228.

3. Batista AC, Silva TA, Chun JH, Lara VS (2002) Nitric oxide synthesis and severity of human periodontal disease. Oral Dis 8, 254-260.

4. Panossian AG, Oganessian AS, Ambartsumian M, Gabrielian ES, Wagner H, Wikman G (1999) Effects of heavy physical exercise and adaptogens on nitric oxide content in human saliva. Phytomedicine 6, 17-26.

5. Konttinen YT, Platts LA, Tuominen S, Eklund KK, Santavirta N, Törnwall K et al. (1997) Role of nitric oxide in Sjögren's syndrome. Arthritis Rheum 40, 875-883.

6. Correia PN, Carpenter GH, Paterson KL, Proctor GB (2010) Inducible nitric oxide synthase increases secretion from inflamed salivary glands. Rheumatology 49, 48-56.

7. Moncada S, Higgs A (1993) The L-arginine-nitric oxide pathway. N Engl J Med 329, 2002-2012.

8. Whittle BJ (1995) Nitric oxide in physiology and pathology. Histochem J 27, 727-737.

9. MacKinnon LT (2000) Special feature for the Olympics: effects of exercise on the immune system: overtraining effects on immunity and performance in athletes. Immunol Cell Biol 78, 502-509.

10. MacKinnnon LT, Jenkins DG (1993) Decreased salivary immunoglobulins after intense interval exercise before and after training. Med Sci Sports Exerc 25, 678-683.

11. Usui T, Yoshikawa T, Orita K, Ueda SY, Katsura Y, Fujimoto $\mathrm{S}$ et al. (2011) Changes in salivary antimicrobial peptides, immunoglobulin A and cortisol after prolonged strenuous exercise. Eur J Appl Physiol 111, 2005-2014.

12. Nieman DC, Johanssen LM, Lee JW, Arabatzis K (1990) Infectious episodes in runners before and after the Los Angeles Marathon. J Sports Med Phys Fitness 30, 316-328.

13. Gleeson M, McDonald WA, Pyne DB, Cripps AW, Francis JL, Fricker PA et al. (1999) Salivary IgA levels and infection 
risk in elite swimmers. Med Sci Sports Exerc 31, 67-73.

14. Gleeson M, Bishop N, Oliveira M, McCauley T, Tauler P, Muhamad AS (2012) Respiratory infection risk in athletes: association with antigen-stimulated IL-10 production and salivary IgA secretion. Scand J Med Sci Sports 22, 410-417.

15. Rahman ZA, Abdullah N, Singh R, Sosroseno W (2010) Effect of acute exercise on the levels of salivary cortisol, tumor necrosis factor-alpha and nitric oxide. J Oral Sci 52, 133-136.

16. Diaz MM, Bocanegra OL, Teixeira RR, Soares SS, Espindola FS (2013) Salivary nitric oxide and alpha-amylase as indexes of training intensity and load. Int J Sports Med 34, 8-13.

17. Li TL, Gleeson M (2004) The effect of single and repeated bouts of prolonged cycling and circadian variation on saliva flow rate, immunoglobulin A and alpha-amylase responses. J Sports Sci 22, 1015-1024.

18. Born DP, Faiss R, Willis SJ, Strahler J, Millet GP, Holmberg HC (2016) Circadian variation of salivary immunoglobulin A, alpha-amylase activity and mood in response to repeated double-poling sprints in hypoxia. Eur J Appl Physiol 116, $1-10$.

19. Harriss DJ, Atkinson G (2013) Ethical standards in sport and exercise science research: 2014 update. Int J Sports Med 34, 1025-1028.

20. Borg GA (1982) Psychophysical bases of perceived exertion. Med Sci Sports Exerc 14, 377-381.

21. Akimoto T, Kumai Y, Akama T, Hayashi E, Murakami H, Soma R et al. (2003) Effects of 12 months of exercise training on salivary secretory IgA levels in elderly subjects. Br J Sports Med 37, 76-79.

22. Alberti A, Bolognini L, Macciantelli D, Caratelli M (2000) The radical cation of $\mathrm{n}, \mathrm{n}$-diethyl-para-phenylendiamine: a possible indicator of oxidative stress in biological samples. Res Chem Intermed 26, 253-267.

23. Trotti R, Carratelli M, Barbieri M (2002) Performance and clinical application of a new, fast method for the detection of hydroperoxides in serum. Panminerva Med 44, 37-40.

24. González D, Marquina R, Rondón N, Rodriguez-Malaver AJ, Reyes R (2008) Effects of aerobic exercise on uric acid, total antioxidant activity, oxidative stress, and nitric oxide in human saliva. Res Sports Med 16, 128-137.

25. Papacosta E, Nassis GP (2011) Saliva as a tool for monitoring steroid, peptide and immune markers in sport and exercise science. J Sci Med Sport 14, 424-434.

26. Chicharro JL, Lucía A, Pérez M, Vaquero AF, Ureña R (1998) Saliva composition and exercise. Sports Med 26, 17-27.

27. Mirvish SS, Reimers KJ, Kutler B, Chen SC, Haorah J, Morris CR et al. (2000) Nitrate and nitrite concentrations in human saliva for men and women at different ages and times of the day and their consistency over time. Eur J Cancer Prev 9, 335-342.

28. Takahama U, Hirota S, Oniki T (2006) Quercetin-dependent scavenging of reactive nitrogen species derived from nitric oxide and nitrite in the human oral cavity: interaction of quercetin with salivary redox components. Arch Oral Biol 51, 629-639.

29. Zetterquist W, Pedroletti C, Lundberg JO, Alving K (1999) Salivary contribution to exhaled nitric oxide. Eur Respir J 13, 327-333.

30. Doel JJ, Hector MP, Amirtham CV, Al-Anzan LA, Benjamin N, Allaker RP (2004) Protective effect of salivary nitrate and microbial nitrate reductase activity against caries. Eur J Oral Sci 112, 424-428.

31. Murase Y, Shimizu K, Tanimura Y, Hanaoka Y, Watanabe K, Kono I et al. (2016) Salivary extracellular heat shock protein 70 (eHSP70) levels increase after 59 min of intense exercise and correlate with resting salivary secretory immunoglobulin A (SIgA) levels at rest. Cell Stress Chaperones 21, 261-269.

32. Ploud D (2005) Nitric oxide and the common cold. Curr Opin Allergy Clin Immunol 5, 37-42.

33. Vianna ML, Dos Santos Rd, Generoso Sde V, Nicoli JR, Martins Fdos S, Nogueira-Machado JA et al. (2013) The role of L-arginine-nitric oxide pathway in bacterial translocation. Amino Acids 45, 1089-1096.

34. Tezuka H, Abe $Y$, Iwata $M$, Takeuchi $H$, Ishikawa $H$, Matsushita M et al. (2007) Regulation of IgA production by naturally occurring TNF/iNOS-producing dendritic cells. Nature 448, 929-933.

35. Ritz T, Trueba AF, Vogel PD, Auchus RJ, Rosenfield D (2018) Exhaled nitric oxide and vascular endothelial growth factor as predictors of cold symptoms after stress. Biol Psychol 132, 116-124.

36. Pannala AS, Mani AR, Spencer JP, Skinner V, Bruckdorfer KR, Moore KP et al. (2003) The effect of dietary nitrate on salivary, plasma, and urinary nitrate metabolism in humans. Free Radic Biol Med 34, 576-584. 